\title{
Strong spectral filtering for a mode-locked similariton fiber laser
}

\author{
Brandon G. Bale ${ }^{1, *}$ and Stefan Wabnitz ${ }^{2}$ \\ ${ }^{1}$ Photonics Research Group, School of Engineering and Applied Science, Aston University, Birmingham B4 7ET, UK \\ ${ }^{2}$ Department of Information Engineering, Via Branze 38, University of Brescia, Brescia 25123, Italy \\ *Corresponding author: b.bale@aston.ac.uk
}

Received May 11, 2010; revised June 8, 2010; accepted June 25, 2010; posted June 28, 2010 (Doc. ID 128310); published July 14, 2010

\begin{abstract}
We propose a novel mode-locked fiber laser design that relies on attracting similariton solutions in fiber amplifiers with normal group-velocity dispersion and strong spectral filtering to compensate increased pulse duration and bandwidth. Stable high-energy, large-bandwidth pulses are obtained that can be linearly compressed, resulting in ultrashort pulses. (C) 2010 Optical Society of America

OCIS codes: $140.4050,140.3538$.
\end{abstract}

Despite much progress in mode-locked fiber lasers, their broader impact has been limited owing to restrictions on pulse energies, which is a consequence of the underlying fiber nonlinearities. The desire for higher energy pulses suggests consideration of fiber lasers that rely on nonconventional pulse shaping mechanisms in the laser cavities [1]. It was shown that high-energy pulses with a parabolic intensity profile and linear frequency chirp can propagate in nonlinear optical fibers with normal group-velocity dispersion (GVD) [2]. These pulses propagate in a self-similar manner, holding certain relations (scaling) between pulse power, duration, and chirp parameter. Recent developments in fiber amplifiers have shown that, in the presence of linear amplification, these solutions, so-called "similaritons," possess the remarkable property of being a global attractor to the system for arbitrary initial conditions [3,4]. In contrast to optical solitons, similaritons can tolerate strong nonlinearity without wave breaking, making them ideal for high-energy pulse propagation in a gain fiber. In the past, fiber lasers based on similaritons have employed self-similar pulse propagation in passive fiber segments [1]. A new, interesting direction was found recently in [ $\overline{5}]$, where both similariton and soliton propagation coexist in the same cavity. In this Letter, we theoretically and numerically investigate a simple fiber laser design whose pulse shaping mechanism relies on the attracting nature of amplifier similaritons, along with strong spectral filtering. The laser can be characterized in terms of solutions to ordinary differential equations for the pulse characteristics in the fiber. In contrast to previous work that considered a distributed Ginzburg-Landau model [6], here the discrete action of the spectral filter is described and found as a function of the filter bandwidth.

We consider a simple loop configuration fiber laser as shown in Fig. 1(a). The laser consists of a ytterbiumdoped fiber operating at $\lambda_{0}=1 \mu \mathrm{m}$ with normal dispersion followed by an output coupler, spectral filter, and saturable absorber. Note that, recently, a similar all-fiber Raman laser has been experimentally realized [7]. Pulse propagation in a rare-earth-doped fiber can be modeled with a normalized equation:

$$
i u_{z}-\frac{1}{2} d u_{t t}+|u|^{2} u=i g\left(u+\nu u_{t t}\right)
$$

where $u$ represents the electric field envelope normalized by the peak field power $P_{0}, t$ represents time in the rest frame of the pulse normalized by $T_{0}$, and $z$ is the propagation distance normalized by the nonlinear length $L_{\mathrm{NL}}=1 /\left(\gamma P_{0}\right)$, where $\gamma=2 \pi n_{2} /\left(\lambda_{0} A_{\text {eff }}\right)$. Here $n_{2}$ is the nonlinear refractive index, $\lambda_{0}$ is the carrier wavelength, and $A_{\text {eff }}$ is the effective fiber area. The normalized GVD coefficient is given by $d=\beta_{2} /\left(\gamma P_{0} T_{0}^{2}\right)$, where $\beta_{2}$ is the fiber GVD coefficient. The dissipative terms on the right-hand side of Eq. (1) represent linear gain as well as a parabolic approximation to the gain profile. Here $\nu=1 /\left(\Omega_{g} T_{0}\right)^{2}$, where $\Omega_{g}$ (gigahertz) is related to the gain bandwidth $\Delta \lambda$ through $\Omega_{g}=\left[2 \pi c / \lambda_{0}^{2}\right] \Delta \lambda$. For high-energy pulses, it is necessary to include gain saturation so that

$$
g=g(z)=\frac{g_{0}}{1+E / E_{s}},
$$

where the energy $E=\int|u|^{2} \mathrm{~d} t, E_{s}=E_{\text {sat }} /\left(P_{0} T_{0}\right)$, and $g_{0}=L_{\mathrm{NL}} G$. Here $G$ (in $\mathrm{dB} / \mathrm{m}$ ) is the linear gain from amplification and $E_{\text {sat }}$ (in nanojoules) is the saturation

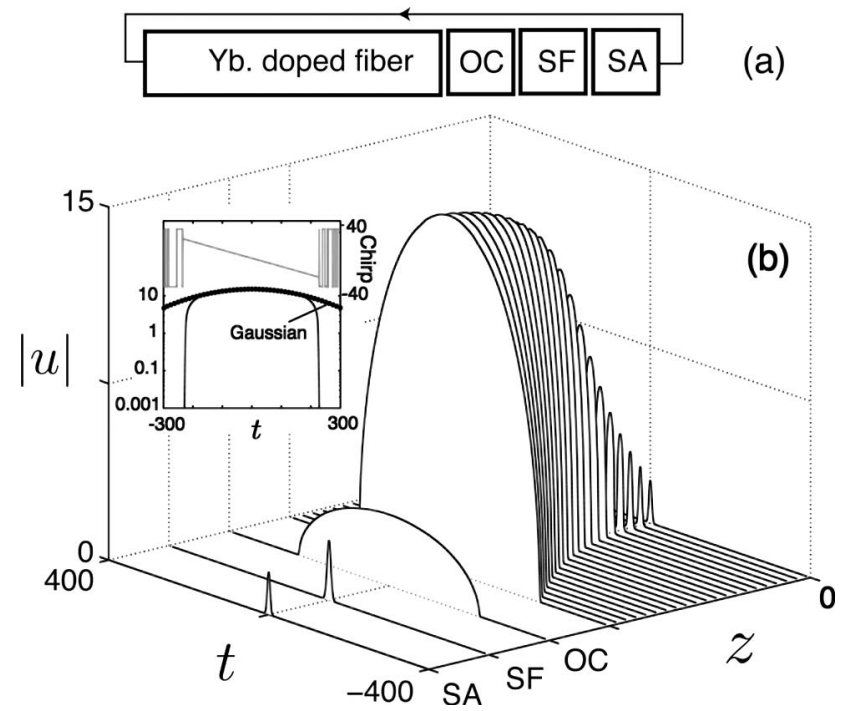

Fig. 1. (a) Laser configuration. (b) Numerical simulation of Eqs. (1)-(3) over one cavity round trip once stable mode locking is obtained. The last three pulses are a result of the discrete transfer functions in Eqs. (3). Inset, intensity (log scale) and chirp of the pulse just before the OC. 
energy of the gain medium. The fiber section of the laser acts as the amplifier. In addition to the amplifier, the discrete actions of the output coupler (OC), spectral filter $(\mathrm{SF})$, and saturable absorber (SA) are required to generate a resonator. In this work, we show that the SF acts as the main pulse shaping mechanism and compensates both the broad pulse duration and bandwidth after the fiber segment. Indeed, pulse shaping by spectral filtering of similaritons was experimentally demonstrated for pulse synthesis [] $]$ and, more recently, in a fiber laser [7]. The discrete elements can be approximated by transfer functions of the form [9]

$$
\begin{gathered}
u_{f}(t)=\rho u_{i}(t), \\
u_{f}(t)=\int_{-\infty}^{\infty} \hat{u}_{i}(\Omega) \times \hat{A}(\Omega) e^{i \Omega t} \mathrm{~d} \Omega, \\
u_{f}(t)=\frac{l_{0}}{1+\left|u_{i}(t)\right|^{2} / P_{s}} \times u_{i}(t),
\end{gathered}
$$

for the OC [Eq. (3a)], SF [Eq. (3b)], and SA [Eq. (3c)]. Here the subscript $i(f)$ denotes the input (output) field, $\rho$ is the output coupling ratio, $\hat{u}(\Omega)$ is the Fourier transform of $u, A(\Omega)$ is the spectral filter profile, $l_{0}$ is the unsaturated loss coefficient, and $P_{s}=P_{\text {sat }} / P_{0}$ is the normalized saturation power, where $P_{\text {sat }}$ (in watts) is the saturation power for the saturable absorber. These discrete elements are critical in returning the pulse to its initial profile, where it will then evolve in the fiber amplifier to its asymptotic state.

Numerical simulations of Eqs. (1) and (2) with the discrete actions of Eqs. (3) show that, for a wide range of parameter space, stable parabolic solutions exist. To illustrate the possible evolution, let us consider a standard fiber that has $\beta_{2}=0.02 \mathrm{ps}^{2} / \mathrm{m}$ at $\lambda_{0}=1 \mu \mathrm{m}$ and nonlinear coefficient $\gamma=0.004(\mathrm{~W} \cdot \mathrm{m})^{-1}$. Let $T_{0}=0.1 \mathrm{ps}$ and $P_{0}=62.5 \mathrm{~W}$, so that the cavity length is normalized by $L_{\mathrm{NL}}=4 \mathrm{~m}$, giving $d=8$. The length of the gain fiber is $5 L_{\mathrm{NL}}$, or $20 \mathrm{~m}$. Further, we consider a gain medium with small signal gain $G=0.375 \mathrm{~dB} / \mathrm{m}$ and saturation energy $62.5 \mathrm{~nJ}$, giving the parameters $g_{0}=1.5$ and $E_{s}=$ $10^{4}$. We set the gain bandwidth to have a width $\Delta \lambda=$ $40 \mathrm{~nm}$, which corresponds to $\nu=0.017$. Finally, the discrete operations in Eqs. (3) have the normalized parameters $\Omega_{f}=0.3$ (filter bandwidth $\Delta \lambda=4 \mathrm{~nm}$ ), $l_{0}=0.9$, $P_{s}=3$ (saturation power $P_{\text {sat }}=187.5 \mathrm{~W}$ ), and $\rho=0.2$. The shape of the spectral filter is taken to be a Gaussian $\hat{A}(\Omega)=\exp \left[-\Omega^{2} /\left(2 \Omega_{f}^{2}\right)\right]$. Figure $1(\mathrm{~b})$ shows the evolution of the simulation over one cavity round trip once stable evolution is achieved. The pulse dynamics act as the global attractor to the system from an arbitrary pulselike initial condition. Figure 2 shows the evolution of the normalized energy $\left(E / \overline{E_{s}}\right)$, peak power $(a)$, rms pulse duration $(\tau)$, and rms bandwidth $(\mathcal{B})$. The pulse evolution in the gain fiber differs from similariton evolution owing to the energy saturation parameter $E_{s}$ and the gain bandwidth parameter $\nu$. For the parameters used in Fig. 1, the gain bandwidth has no effects on propagation, and pulse evolution depends on the ratio $E / E_{s}$. For $E / E_{s} \ll 1$, the pulse evolution is self-similar, as for similariton (constant gain) amplification [3]. As $E / E_{s} \sim 1$, the peak power le- vels off while the pulse duration increases linearly. From Figs. 2(c) and 2(d), we see that the evolution in the laser exhibits large temporal and spectral breathing. The spectral filter compensates the pulse duration and bandwidth. Thus, the main pulse shaping mechanism relies on the attracting nature of parabolic pulse formation in the gain fiber, which is then compensated by a strong spectral filter. Although the saturable absorber is not significant in shaping the pulse, it improves the stability of the laser by attenuating low amplitude wings that can grow over many cavity round trips. The output is both temporally and spectrally very broad and has pulse energy of $\sim 7 E_{\text {sat }}$, corresponding to $\sim 0.5 \mu \mathrm{J}$, which is reaching the limits of state-of-the-art high-energy fiber lasers [10]. Since the output pulse has a linear chirp and a broad bandwidth, it can be efficiently linearly compressed outside the laser cavity giving high-energy, ultrashort pulses.

To gain insight into the pulse evolution in the gain fiber, we look for self-similar solutions [2]:

$$
u(\boldsymbol{z}, t)=\sqrt{a(\boldsymbol{z})} f\left(\frac{t}{\tau(\boldsymbol{z})}\right) e^{i C(\boldsymbol{z}) t^{2}+i q(\boldsymbol{z})},
$$

where $a$ is the peak power, $\tau$ is the pulse duration, $C$ is the chirp parameter, $q$ is the phase parameter, and $f$ is the functional form of the pulse. Inserting Eq. (4) into Eq. (1)
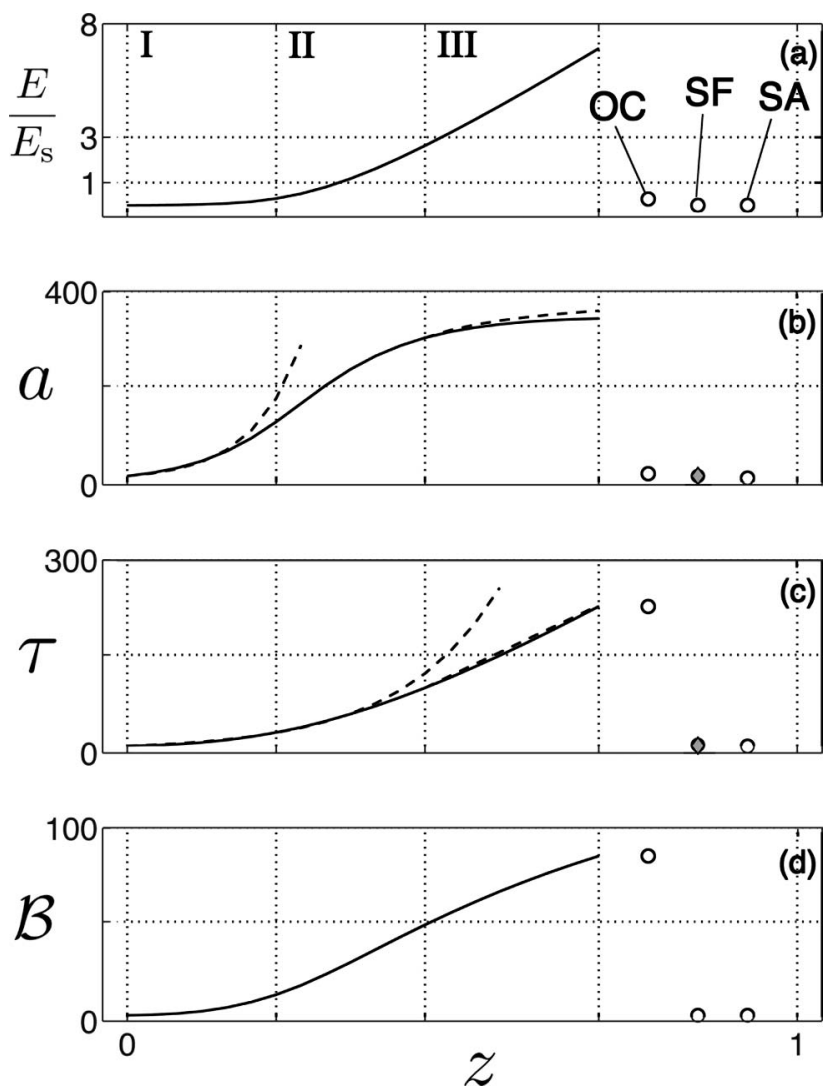

Fig. 2. Evolution of the pulse (a) normalized energy, (b) peak power, (c) duration, and (d) bandwidth for the simulation shown in Fig. 1(b) (solid curves) and analytical results (dashed curves). The last three points correspond to the discrete elements (shaded diamond is for analytic). Regions I and III correspond to the "similariton" and "saturated" regimes, respectively. 
$(\nu=0)$, letting $f(t)=\sqrt{1-t^{2}}$, and assuming $1 / \tau^{2} \ll 1$, gives the ordinary differential equations

$$
\begin{gathered}
a_{z}=2 d C a+\frac{2 g_{0} a}{1+4 a \tau /\left(3 E_{s}\right)}, \\
\tau_{z}=-2 d C \tau, \\
C_{z}=2 d C^{2}-\frac{a}{\tau^{2}},
\end{gathered}
$$

and $q_{z}=a^{2}(z)$. In the limit of $E \ll E_{s}$ ("similariton regime"), Eqs. (5) have the self-similar solutions $a(\boldsymbol{z})=a_{0} \exp \left[4 g_{0} \boldsymbol{z} / 3\right], \tau(\boldsymbol{z})=\tau_{0} \exp \left[2 g_{0} \boldsymbol{z} / 3\right]$, and $C(\boldsymbol{z})=$ $-g_{0} /(3 d)$, with the initial conditions $a_{0}$ and $\tau_{0}$ given in terms of the initial energy $\mathcal{E}_{0}: a_{0}=\left[9 d /\left(2 g_{0}^{2}\right)\right]^{-1 / 3} \mathcal{E}_{0}^{2 / 3}$ and $\tau_{0}=\left[9 d /\left(2 g_{0}^{2}\right)\right]^{1 / 3} \mathcal{E}_{0}^{1 / 3}$ [3]. When the pulse energy is much greater than the saturation energy $1 \ll E / E_{s}$ ("saturation regime"), the square of the pulse duration is much larger than the peak power. Neglecting the $a / \tau^{2}$ term in Eq. (5c), the system in Eqs. (5) has the solutions $\quad a(z)=\left(a_{0}+3 g_{0} E_{s} z /\left(2 \tau_{0}\right)\right) /\left(1-2 \bar{d} C_{0} z\right), \quad \tau(z)=$ $\tau_{0}\left(1-2 d C_{0} z\right)$, and $C(\boldsymbol{z})=C_{0} /\left(1-2 d C_{0} z\right)$, where $a_{0}, \tau_{0}$, and $C_{0}(<0)$ are the initial values of the pulse parameters upon entering this regime. In contrast to the similariton regime, the peak power levels off and the pulse duration increases linearly with distance in the saturation regime. The analytic solutions in both the "similariton" and "saturation" regimes are plotted in Fig. $\underline{2}$ and show good agreement with simulations.

The effect of the discrete elements can be viewed as a transformation on the pulse parameters $\left(a_{i}, \tau_{i}, C_{i}\right) \rightarrow$ $\left(a_{f}, \tau_{f}, C_{f}\right)$ [9]. The output coupler is simply a scalar multiplication on the power $a_{f}=\rho^{2} a_{i}$. In general, the spectral filter will act on a highly chirped parabolic pulse of the form (4). Approximating the Fourier transform of Eq. (4) by the use of a stationary phase integral, it is easy to show that $\hat{u}(\Omega) \sim \sqrt{a \pi /(2 i C)} \times \sqrt{1-[\Omega /(2 C \tau)]^{2}} \times$ $\exp \left[-i \Omega^{2} /(4 C)\right]$. Because the pulse is broad and highly chirped, one can expand the parabolic function using $\sqrt{1-x^{2}} \sim 1-x / 2+\ldots$ Assuming a strong spectral filter so that the bandwidth of the spectral filter is narrow compared to the pulse bandwidth, one can approximate $\hat{u}$ with only the first term in the expansion. Multiplying by the filter function $\hat{A}(\Omega)$ and taking the inverse transform gives the modified pulse parameters. Using the same Gaussian spectral filter function as in the simulation in Fig. 1(b), the result of such a calculation is a Gaussian function of the form $u_{f}(t)=\sqrt{a_{f}} \exp \left[-\left(t / \tau_{f}\right)^{2}\right.$ $\left.+i C_{f} t^{2}\right]$, where

$$
a_{f}=\frac{1}{4}\left[\frac{\Omega_{f}^{2}}{C_{i}^{2}+4 \Omega_{f}^{4}}\right] a_{i},
$$

$$
\begin{aligned}
\tau_{f} & =\sqrt{\frac{\left(4 C_{i}^{2}+\Omega_{f}^{4}\right)}{2 C_{i}^{2} \Omega_{f}^{2}},} \\
C_{f} & =\left[\frac{\Omega_{f}^{4}}{4 C_{i}^{2}+\Omega_{f}^{4}}\right] C_{i} .
\end{aligned}
$$

Thus we see that, for strong spectral filter $\left(\Omega_{f}<1\right)$, the pulse power, duration, and chirp parameter are decreased $\propto \Omega_{f}^{2}, \Omega_{f}^{-1}, \Omega_{f}^{4}$, respectively. When applying Eqs. (6) to the pulse resulting from the output coupler in the simulation in Fig. 2, the values of $a_{f}=8.9, \tau_{f}=$ 6.7 are in good agreement with the values from simulation $a_{f}=8.6, \tau_{f}=7.5$ [diamonds overlapping circles in Figs. 2(b) and 2(c)].

In general, extending the length of the fiber amplifier will allow for higher pulse energies with broader bandwidths. However, numerical simulations show that, if the pulse bandwidth approaches the gain bandwidth, both the temporal profile and the chirp will be reshaped. Extending the cavity so as to support parabolic pulse propagation, as well as increasing the pulse bandwidth past the gain bandwidth, will be reported elsewhere.

In conclusion, we have theoretically and numerically investigated a simple fiber laser design whose pulse shaping mechanism relies on the attracting nature of amplifier similaritons, which is then compensated by a strong spectral filtering. The pulse evolution can be completely characterized by solutions to the ordinary differential equations for the pulse characteristics in the fiber, along with scalar transfer functions for the spectral filter. Because of the pulse structure, efficient compression outside the resonator leads to high-energy ultrashort pulses.

B. G. Bale would like to acknowledge insightful discussions with Frank Wise and support by the UK Engineering and Physical Sciences Research Council (EPSRC) (grant no. EP/FO2956X/1) and the British and Italian Partnership Programme 2009-2010.

\section{References}

1. F. W. Wise, A. Chong, and W. H. Renninger, Laser Photon. Rev. 2, 58 (2008).

2. D. Anderson, M. Desaix, M. Karlsson, M. Lisak, and M. L. Quiroga-Teixeiro, J. Opt. Soc. Am. B 10, 1185 (1993).

3. M. E. Fermann, V. I. Kruglov, B. C. Thomsen, J. M. Dudley, and J. D. Harvey, Phys. Rev. Lett. 84, 6010 (2000).

4. V. I. Kruglov and J. D. Harvey, J. Opt. Soc. Am. B 23, 2541 (2006).

5. B. Oktem, C. Ülgüdür, and F. Ö. Ilday, Nat. Photon. 4, 307 (2010).

6. J. F. Zhang, L. Wu, and L. Li, Phys. Rev. A 78, 055801 (2008).

7. C. Aguergaray, D. Méchin, V. Kruglov, and J. D. Harvey, Opt. Express 18, 8680 (2010).

8. C. Finot and G. Millot, Opt. Express 12, 5104 (2004).

9. B. G. Bale, S. Boscolo, J. N. Kutz, and S. K. Turitsyn, Phys. Rev. A 81, 033828 (2010).

10. B. Ortac, M. Baumgartl, J. Limpert, and A. Tunnermann, Opt. Lett. 34, 1585 (2009). 